\title{
Decreased Indian hedgehog signaling activates autophagy in endometriosis and adenomyosis
}

\author{
Yingying Zhou ${ }^{1}$, Yangying Peng², Qingqing Xia', Dewen Yan², Huiping Zhang², Lingmin Zhang , \\ Ying Chen ${ }^{1}$, Xiumin Zhao $^{2}$ and Jie $\mathrm{Li}^{1}$ \\ ${ }^{1}$ Department of Laboratory Medicine, Huangyan Hospital of Wenzhou Medical University, Taizhou First People's \\ Hospital, Taizhou, Zhejiang Province, China and '2Department of Obstetrics and Gynecology, Huangyan Hospital of \\ Wenzhou Medical University, Taizhou First People's Hospital, Taizhou, Zhejiang Province, China \\ Correspondence should be addressed to X Zhao or J Li; Email: 40676951@qq.com or liyijie12580@126.com
}

\begin{abstract}
Indian hedgehog (Ihh) signaling regulates endometrial receptivity and is an indispensable mediator of embryonic implantation. Hedgehog signaling is known to regulate autophagy, and aberrant regulation of autophagy is critically implicated in the pathogenesis of endometriosis and adenomyosis. However, potential dysregulation of Ihh signaling and its role in autophagy modulation in these diseases remain obscure. In this study, we found that components of Ihh signaling were significantly decreased, whereas the autophagy marker protein, LC3BII, was significantly increased in endometrial tissues of women with endometriosis or adenomyosis. Inhibition of Ihh signaling with the small-molecule inhibitor GANT61 or Gli1 silencing in primary endometrial stromal cells increased autophagic activity, as measured by LC3 turnover assay and tandem mCherry-eGFP-LC3B fluorescence microscopy. Furthermore, we observed that GANT61 treatment significantly attenuated hydrogen peroxide-induced cell death, whereas disruption of autophagy with chloroquine diminished this effect. Collectively, these findings reveal that lhh signaling is suppressed in endometrial tissues of patients with endometriosis or adenomyosis. This abnormal decrease may contribute to endometrial autophagy activation, which may promote aberrant survival of endometrial cells in ectopic sites in these two gynecological diseases.
\end{abstract}

Reproduction (2021) 161 99-109

\section{Introduction}

Endometriosis and adenomyosis are common, refractory gynecological disorders affecting $10-19.5 \%$ of fertile women worldwide (Choi et al. 2015, Garcia-Solares et al. 2018, Zondervan et al. 2020). They present clinically with chronic progressive pelvic pain, dysmenorrhea, and infertility. Endometriosis is characterized by the presence of functional endometrial tissue outside the uterine cavity, whereas adenomyosis is defined by the presence of ectopic endometrial glands and stroma within the myometrium (Vannuccini et al. 2017, Kobayashi et al. 2020, Zondervan et al. 2020). These diseases exhibit differences in risk factors, some clinical features, pathological characteristics, and pathogenesis. However, they also share many similarities, including estrogen dependency, progesterone resistance, and multiple cellular and molecular aberrations (Vannuccini et al. 2017, Kobayashi et al. 2020, Zhu et al. 2016). For many years, adenomyosis was considered a specific form of endometriosis called endometriosis interna (Vannuccini et al. 2017, Che et al. 2020). Although endometriosis and adenomyosis cause severe symptoms in patients, they are generally believed to be chronic benign uterine diseases. However, ectopic endometrial cells also have certain malignant biological behaviors, such as aggressive invasion, migration, unrestrained proliferation, and reduced susceptibility to apoptosis (Liu et al. 2017, 2019b, Che et al. 2020). These features are important for the pathogenesis of endometriosis and adenomyosis.

Autophagy is a dynamic, multistage process that includes the formation of the phagophore, the autophagosome, and theautolysosome. Thephagophore is the initial sequestering double-membrane compartment, which expands and surrounds the targeted cytoplasmic material to form the autophagosome. The completion of autophagic flux occurs when autophagosomes fuse with lysosomes to generate autolysosomes, resulting in the degradation of the contents (Klionsky et al. 2016, Yim \& Mizushima 2020). Autophagy is essential for cellular homeostasis and adaptation to stress (Leidal et al. 2018, Mizushima 2018). Autophagy in adenomyosis patients is poorly understood, and its role in endometriosis remains controversial (Allavena et al. 2015, Mei et al. 2015, Matsuzaki et al. 2018, Zhan et al. 2018, Liu et al. 2019b). However, recent evidence has demonstrated that autophagic dysregulation may be implicated in the pathogenesis of endometriosis and adenomyosis (Ren et al. 2010, Allavena et al. 2015, Mei et al. 2015, 
Matsuzaki et al. 2018, Zhan et al. 2018, Liu et al. 2019b). Despite a significant increase in our knowledge regarding autophagy, the mechanism responsible for its regulation remains largely unknown in endometrial tissues of women with endometriosis or adenomyosis.

The hedgehog $(\mathrm{Hh})$ signaling pathway regulates embryonic development, cell proliferation, differentiation, and cell-cell communication (Lee et al. 2006, Wang et al. 2013). Hh ligands, including Indian $\mathrm{Hh}$ (Ihh), Sonic Hh, and Desert Hh, bind to patched receptors (Ptch1 and Ptch2), subsequently abolish Ptchinduced smoothened (Smo) inhibition, and activate the glioma zinc finger transcriptional factors (Gli1, Gli2, and Gli3) (Wang et al. 2013). Ihh is essential for normal progesterone action, estrogen signaling, and uterine epithelium and stroma cellular communication (Lee et al. 2006, Franco et al. 2010, Yoo et al. 2017). Studies have demonstrated that the expression of Ihh and Gli1 may be decreased in endometriosis (Smith et al. 2011, Yoo et al. 2017). In contrast, other studies have indicated that $\mathrm{Hh}$ signaling may be enhanced in endometriosis ( $\mathrm{He}$ et al. 2018, Liu et al. 2019a). Currently, there is no relevant study with respect to the role of Ihh signaling pathway in adenomyosis. Therefore, the regulation of Ihh signaling in endometriosis and adenomyosis requires further clarification.

Hh signaling is intimately associated with autophagy regulation; however, whether $\mathrm{Hh}$ pathway inhibits or activates autophagy may be context-dependent. Accumulating evidence has demonstrated that $\mathrm{Hh}$ signaling negatively regulates autophagy (JimenezSanchez et al. 2012, Wang et al. 2013). In some cell types, such as hippocampal neurons and vascular endothelial cells, Hh signaling promotes autophagy (Li et al. 2012, Petralia et al. 2013). To our knowledge, it has not been determined whether Ihh signaling is involved in the autophagic regulation of endometrial cells in endometriosis or adenomyosis patients.

In this study, we determined whether autophagy and Ihh signaling are aberrantly altered in the endometria of patients with endometriosis or adenomyosis and elucidated the role of Ihh pathway in endometrial autophagy regulation in these two diseases.

\section{Materials and methods \\ Ethical approval and tissue collection}

This study was approved by the Institutional Ethics Committee of Taizhou First People's Hospital (approval number: 2019KY006-01). All procedures were performed in accordance with the Declaration of Helsinki and the relevant guidelines and regulations. Written informed consent was obtained from all patients before endometrial tissue sample collection.

Patients of reproductive age (23-50 years old) with leiomyoma, endometriosis, or adenomyosis who attended the Department of Obstetrics and Gynecology in our hospital were recruited for this study. None of the study participants had taken any contraceptives, used intrauterine devices, or received hormonal therapy for at least 3 months prior to surgery. Endometrial samples were collected during the proliferative stage of the menstrual cycle, which was confirmed by histological criteria. Control endometrial tissues (Con-E) were collected from patients with leiomyoma $(n=15)$ who underwent laparoscopic myomectomy. We collected 11 cases of ovarian endometriotic tissues (EDM-ecE) from women who underwent ovarian cystectomy by laparoscopy. Specimens of EDM-ecE were carefully stripped from the inner wall of the ovarian endometriotic cysts. Ten cases of eutopic endometria (EDM-euE) were obtained from another group of patients with ovarian endometriosis. Samples from patients with adenomyosis, including the eutopic endometrium (ADM-euE) and the corresponding ectopic lesions of adenomyosis (ADMecE), were collected from patients $(n=18)$ who underwent hysterectomy. These samples were used for quantitative real-time PCR (qPCR), immunohistochemical staining, and Western blot analysis. However, the ectopic endometrial tissue of adenomyosis usually presents as small focal or diffuse lesions. Therefore, we were unable to obtain sufficient ectopic endometrium for qPCR and immunoblot detection. An additional 14 cases of eutopic endometrial tissue from patients with adenomyosis (6 cases) and endometriosis (8 cases) were collected for isolation and cultivation of endometrial stromal cells.

\section{Isolation and identification of endometrial stromal cells}

Eutopic endometrial stromal cells (EuESCs) were isolated and purified as previously described (Liu et al. 2017). Briefly, fresh endometrial tissues were washed with PBS to remove blood, and then the tissues were minced with sterile surgical scissors and digested in DMEM/F12 medium (SH30023.01, Sigma-Aldrich) containing $2.5 \mathrm{mg} / \mathrm{mL}$ of type I collagenase (17100017, Gibco, Thermo Fisher Scientific) at $37^{\circ} \mathrm{C}$ for 90 min. EuESCs were obtained from two sequential filtrations with a 100- $\mu \mathrm{m}$ and a $40-\mu \mathrm{m}$ cell strainer (15-1100 and 15-1040, Biologix) and were cultured in DMEM/F12 supplemented with $10 \%$ fetal bovine serum (FBS) (RY-F22-05, Roya Biotechnology Company, Lanzhou, Gansu, China) and 1\% (v/v) penicillin/ streptomycin (10378016, Gibco) in a humidified atmosphere of $5 \% \mathrm{CO}_{2}$ at $37^{\circ} \mathrm{C}$ overnight. The medium was then discarded, and the cells were gently washed with PBS to remove blood cells and debris. The purity of EuESCs in our experiment was $>95 \%$, as determined by positive immunostaining for vimentin and negative staining for cytokeratins-19 (Choi et al. 2015, Che et al. 2020). Second and third passages of cells were used for subsequent experiments.

\section{In vitro experiments}

EuESCs were seeded into six-well cell culture plates with DMEM/F12 supplemented with $10 \%$ FBS (complete medium) and cultured for $24 \mathrm{~h}$. The cells were then treated with a smallmolecule inhibitor of Hh signaling, GANT61 (0-20 $\mu \mathrm{M}$; S8075, Selleck Chemicals), in serum-free DMEM/F12 for $24 \mathrm{~h}$. For the LC3 turnover assay (Mizushima et al. 2010), which is one of 
the principal methods to monitor autophagic flux, EuESCs were cultured in serum-free medium with $10 \mu \mathrm{M}$ GANT61 for $24 \mathrm{~h}$ in the absence or presence of 3-methyladenine (3-MA) ( 2.5 mM; S2767, Selleck) or chloroquine (CQ) $(10 \mu M$; S4157, Selleck). To clarify the role of autophagy in oxidative stressinduced cell death, EuESCs were treated with $10 \mu \mathrm{M}$ GANT61 for $24 \mathrm{~h}$ in the absence or presence of $10 \mu \mathrm{M} \mathrm{CQ}$, the medium was then removed, and the cells were exposed to $200 \mu \mathrm{M}$ of hydrogen peroxide $\left(\mathrm{H}_{2} \mathrm{O}_{2}\right)$ in serum-free medium for $2 \mathrm{~h}$. The cells were washed twice with PBS and harvested for Western blotting analysis.

\section{qPCR}

EuESCs were directly scraped and harvested using RNAiso Plus reagent (9109, TakaRa), and tissues were homogenized with this reagent using a glass tissue grinder on ice. Total RNA was extracted and purified according to the manufacturer's instructions. cDNA was synthesized using a PrimeScript ${ }^{\mathrm{TM}}$ RT Master Mix Kit (RR036A, TakaRa). Quantitative PCR was performed on an ABI Prism ${ }^{\circledR} 7500$ real-time PCR detection system (Applied Biosystems, Thermo Fisher Scientific) using a TB Green ${ }^{\circledR}$ Premix Ex Taq ${ }^{\text {TM }}$ (Tli RNaseH Plus) kit (RR420A, TakaRa). The following primers were used for the qPCR analysis: Ihh, 5'-CATTGAGACTTGACTGGGCAAC-3' and 5'-AGAGCAGGCTGAGTTGGGaGTCGC-3'; Ptch 1, 5'-GGCTCCCTCATCTCGTAAC-3' and 5'-ACTATGCTGTGGGTATTTCTGG-3'; Smo, 5'-GCTACTTCCTCATCCGAGGAGTCA-3' and 5'-GGCGCAGCATGGTCTCGTT-3'; Gli1, 5'-TCCTACCAGAGTCCCAAGTT- $3^{\prime}$ and 5'-CCCTATGTGAAGCCCTATTT- $3^{\prime}$; and GAPDH 5' $^{\prime}$-TGACGCTGGGGCTGGCATTG- ${ }^{\prime}$ and 5'-GGCTGGTGGTCCAGGGGTCT-3'. Each sample was measured in triplicate, and the reactions without any CDNA template were used as negative controls. The $2^{-\Delta \Delta C t}$ method was used to calculate the expression of each target gene, as previously described (Livak \& Schmittgen 2001), and GAPDH served as a loading control.

\section{Immunohistochemical analysis}

Immunohistochemical staining was performed on thin paraffin sections with the following primary antibodies: mouse anti-Gli1 (1:100; 66905-1-lg, Proteintech) and rabbit anti-Gli2 (1:400; A16864, ABclonal technology). Sections were dewaxed and rehydrated in graded ethanol (80-100\%). Antigen retrieval was achieved using an EDTA antigen retrieval solution $(\mathrm{pH} 8.0)$ in a pressure cooker for $2.5 \mathrm{~min}$ at full pressure. Sections were then treated twice with $3 \%$ $\mathrm{H}_{2} \mathrm{O}_{2}$ for 5 min each to eliminate endogenous peroxidase activity and then blocked with normal goat serum for $30 \mathrm{~min}$ at room temperature. Sections were subsequently incubated with primary antibodies overnight at $4{ }^{\circ} \mathrm{C}$. After rinsing three times with PBS, sections were incubated with horseradish peroxidase (HRP)-conjugated secondary antibody (ready-touse; DS-0004, ZSGB-BIO Company, Beijing, China) for 30 min. Finally, all slides were incubated with DAB Substrate and counterstained in hematoxylin before they were dehydrated and mounted. Dark-brown staining indicated a positive reaction. Results were imaged using an inverted microscope
(Olympus, IX53). Quantitative analysis was performed on three to five random fields at $400 \times$ magnification for each endometrial slice. The intensity of the dark-brown staining was analyzed using ImageJ software (NIH).

\section{Western blot analysis}

To prepare protein extracts, tissues were homogenized in radioimmunoprecipitation assay lysis buffer (R0020, Solarbio, Beijing, China) containing $1 \%$ protease inhibitor cocktail (FD1001, Fude Biological Technology, Hangzhou, Zhejiang, China) using a glass tissue grinder on ice. EuESCs were directly scraped in the lysis buffer to obtain cellular proteins. Samples were subjected to electrophoresis with SDS-PAGE denaturing gels, and the separated proteins were transferred to polyvinylidene difluoride membranes (146588, Millipore). The membranes were blocked in $5 \%$ nonfat milk at room temperature for $1 \mathrm{~h}$ and then incubated overnight with primary antibodies at $4{ }^{\circ} \mathrm{C}$. Subsequently, the membranes were washed with Tris-buffered saline containing $0.1 \%$ Tween-20 and incubated for $1 \mathrm{~h}$ with the corresponding HRP-conjugated secondary antibodies (1:1000; A0216 and A0208, Beyotime) at room temperature. Western ECL Substrate (1705060, Clarity ${ }^{\mathrm{TM}}$, Bio-Rad) was used for protein band visualization, and the bands were detected and analyzed with the ChemiDox ${ }^{\mathrm{TM}}$ XRS+ system (Bio-Rad) and Image Lab ${ }^{\text {TM }}$ software (Bio-Rad). The following primary antibodies were used for these experiments: rabbit antiLC3B (1:1000; \#3868, Cell Signaling Technology (CST)), rabbit anti-Beclin1 (1:1000; \#3495, CST), mouse anti-Gli1 (1:1000; 66905-1-lg, Proteintech), rabbit anti-Gli2 (1:1000; A16864, Proteintech), rabbit anti-GAPDH $(1: 1000 ; \# 2118, C S T)$, and mouse anti- $\beta$-actin (1:1000; 60008-1-lg, Proteintech).

\section{Cytotoxicity analysis}

EuESCs $\left(5 \times 10^{3}\right.$ cells/well) were cultured in 96 -well cell culture plates with complete medium for $24 \mathrm{~h}$. After the supernatant was aspirated, cells were treated with GANT61 $(0-20 \mu \mathrm{M})$ in serum-free DMEM/F12 for $24 \mathrm{~h}$. The medium was then discarded, and $100 \mu \mathrm{M}$ of DMEM/F12 with $10 \%$ Cell Counting Kit-8 (CK04, Dojindo) was added to each well and incubated at $37^{\circ} \mathrm{C}$ for $1 \mathrm{~h}$. The absorbance (optical density) at $450 \mathrm{~nm}$ was measured using a microplate spectrophotometer (Multiskan FC, Thermo Fisher Scientific). Cell viability measurements are presented as the proportion of control optical density.

\section{Nuclear morphology analysis after cell staining with DAPI}

EuESCs $\left(3 \times 10^{4}\right.$ cells/well) were cultured in 24-well cell culture plates for $24 \mathrm{~h}$. The cells were then incubated with GANT61 $(10 \mu \mathrm{M})$ alone or in combination with CQ $(10 \mu \mathrm{M})$ in serum-free DMEM/F12 for $24 \mathrm{~h}$. Subsequently, the medium was removed, and the cells were exposed to $200 \mu \mathrm{M} \mathrm{H} \mathrm{H}_{2} \mathrm{O}_{2}$ in the serum-free medium for $2 \mathrm{~h}$. EuESCs were then fixed with $4 \%$ paraformaldehyde for $15 \mathrm{~min}$ at room temperature, followed by incubation for $5 \mathrm{~min}$ with $1 \mu \mathrm{g} / \mathrm{mL}$ DAPI (D9542, Sigma-Aldrich) in the darkness. Cell nuclei were observed using an inverted fluorescence microscope (Olympus, 
IX53). Generally, normal nuclei are round, clear-edged, and uniformly stained, whereas apoptotic nuclei exhibit irregular edges around the nucleus and are more intensely stained and pyknotic. For apoptotic cell analysis, at least five views from each well were imaged, and the ratio of condensed nuclei to total nuclei was calculated and expressed as the apoptosis rate for each group.

\section{Tandem mCherry-eGFP-LC3B fluorescence microscopy}

Recombinant adenovirus vectors packing a tandem monomeric mCherry-eGFP-tagged LC3B construct for autophagic flux monitoring were generated by Vigene Biosciences (Jinan, Shandong, China). According to the manufacturer's instructions, $5 \times 10^{5}$ EuESCs were seeded in a $35-\mathrm{mm}$ glass-bottomed dish (D35C4-20-1-N, Cellvis) and maintained for $24 \mathrm{~h}$ in complete medium. At approximately $60-70 \%$ confluence, the cells were cultured in $500 \mu \mathrm{L}$ of serum-free DMEM/F12 containing $2 \mu \mathrm{L}$ of recombinant adenovirus $\left(3.2 \times 10^{8} \mathrm{pfu} / \mathrm{mL}\right)$. After $24 \mathrm{~h}$, the medium was discarded, and the cells were treated with $10 \mu \mathrm{M}$ GANT61 in DMEM/F12 for $24 \mathrm{~h}$. The cells were then observed and imaged using confocal microscopy (Olympus, FV3000). Autophagic flux was determined by evaluating the number of mCherry+eGFP- and mCherry+eGFP+ puncta (puncta/cell were counted).

\section{SiRNA transfection}

Gli1 siRNA (5'-CCGAAGGACAGGUAUGUAATT-3') and a negative control (NC) siRNA (5'-GCGACGAUCUGCCUAAGAUdTdT-3') were designed and synthesized by GenePharma (Shanghai, China). EuESCs $\left(5 \times 10^{5}\right.$ cells/well) were cultured in 6-well cell culture plates in complete medium for $24 \mathrm{~h}$. According to the manufacturer's instructions, si-Gli1 or NC (100 $\mathrm{nM}$ ) working solutions were prepared with Lipofectamine ${ }^{\mathrm{TM}}$ 3000 (L3000001, Thermo Fisher Scientific) and Opti-MEM ${ }^{\mathrm{TM}}$ (31985070, Gibco). At approximately 60-70\% confluence, EuESCs were incubated with siRNA working solution for $6 \mathrm{~h}$. Then the cells were maintained in complete medium for $24 \mathrm{~h}$. Afterward, the cells were cultured in serum-free DMEM/F12 in the absence or presence of $10 \mu \mathrm{M}$ CQ for $24 \mathrm{~h}$.

\section{Statistical analysis}

All data were expressed as means \pm S.E.M. For statistical analysis, the Student's t-test was used for data with only two groups; the one-way ANOVA followed by Tukey's multiple comparisons test was used for data containing more than two groups. $P<0.05$ was considered statistically significant.

\section{Results}

\section{Ihh signaling is inhibited in endometrial tissues of endometriosis patients}

We evaluated the expression levels of Ihh signaling pathway components in eutopic and ectopic endometrial tissues isolated from patients with endometriosis by
qPCR. As shown in Fig. 1A, B, C and D, the mRNA levels of Ihh, Ptch1, Smo, and Gli1 tended to decrease in both eutopic and ectopic endometrial tissues. Though there was no statistically significant difference between control and eutopic endometrial tissues, inhibition of Ihh, Ptch1, and Smo expression in ectopic tissues was evident (Fig. 1A, B and C). The transcription factor Gli1, which functions as an amplifier of the pathway (Dai et al. 1999, Ruiz I Altaba 1999, Gorojankina 2016, Shi \& Long 2017), was significantly suppressed in both eutopic and ectopic endometrium (Fig. 1D). Gli2 predominantly acts as a transcriptional activator and is considered a marker of Ihh pathway activation (Jimenez-Sanchez et al. 2012, Shi \& Long 2017). Therefore, we further analyzed the expression levels of Gli1 and Gli2 proteins in endometrial tissues from women with endometriosis. Western blot analysis revealed that Gli1 expression tended to decrease in the eutopic endometrium and was significantly inhibited in the ectopic endometrium (Fig. $1 \mathrm{E}$ and $\mathrm{F}$ ). In addition, Gli2 expression was also markedly suppressed in ectopic endometriotic tissues, whereas there was no significant difference in Gli2 expression between the eutopic and control endometrium (Fig. 1E
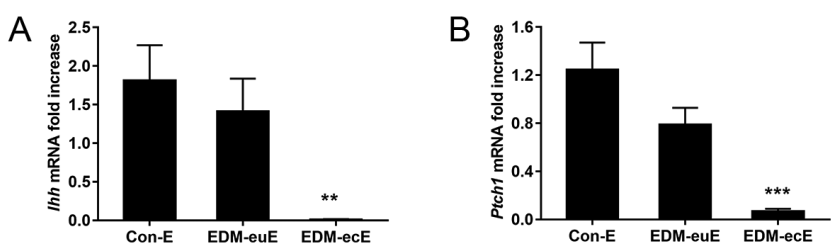$$
\text { C }
$$
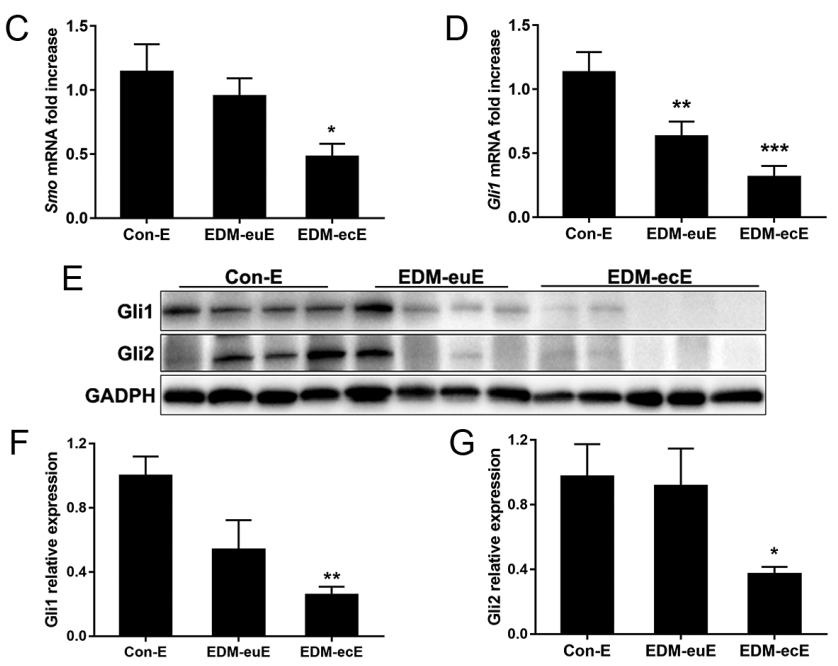

Figure 1 Ihh signaling is inhibited in endometrial tissues of endometriosis patients. (A, B, C and D) Ihh, Ptch 1, Smo and Gli1 mRNA expression levels were assessed by qPCR in Con-E, EDM-euE and EDM-ecE; $n=10$. (E) Representative immunoblots of Gli1 and Gli2 in Con-E, EDM-euE and EDM-ecE. (F and G) Densitometric quantification of Gli1 and Gli2 in Con-E, EDM-euE and EDM-ecE; $n=10$ or 11 . Quantitative results are expressed as means \pm S.E.M.; ${ }^{*} P$ $<0.05,{ }^{* *} P<0.01,{ }^{* * *} P<0.001$. Con-E: endometrium from patients with leiomyoma; EDM-euE and EDM-ecE: eutopic and ectopic endometrial tissues of endometriosis patients, respectively. 
and G). These data indicate that Ihh signaling is inhibited in the endometrium of endometriosis patients.

\section{Ihh signaling is inhibited in endometrial tissues of women with adenomyosis}

We next examined the expression of Ihh pathway components in eutopic endometrial tissues of patients with adenomyosis. We found that the expression levels of Ihh, Ptch1, Smo, and Gli1 in eutopic endometrium were decreased by approximately $50 \%$ compared with that of the control endometrium (Fig. 2A, B, C and D). Immunohistochemical staining of Gli1 revealed that it was predominantly localized in the cytoplasm of glandular and stromal cells, and the staining levels were decreased either in eutopic endometrium or in ectopic endometrial cells compared with the control group (Fig. $2 \mathrm{E}$ and F). Gli2 was observed in the cytoplasm and nuclei of glandular and stromal cells in the functional layer of the endometrium (Fig. 2G). It was also predominantly localized to the cytoplasm of glandular and stromal cells in the basal layer of the endometrium and ectopic tissues (Fig. 2G). Furthermore, Gli2 expression was significantly decreased in the basal layer of control endometrium (Con-E-B) and the basal layer of eutopic tissues from patients with adenomyosis (ADM-euE-B) compared with the respective endometrial functional layer (Con-E-F or ADM-euE-F) (Fig. 2G and H). Although no significant differences were observed between the control and the eutopic endometrial tissues, Gli2 expression was somewhat decreased in tissues of adenomyosis patients and was significantly inhibited in ectopic tissues (ADMecE) compared with Con-E-F or ADM-euE-F (Fig. 2G and $\mathrm{H}$ ). To further examine Gli1 and Gli2 expression in endometria from patients with adenomyosis, we quantified these proteins in eutopic tissues by Western blot analysis. The data indicated that Gli1 and Gli2 were significantly downregulated in women with adenomyosis (Fig. 2I, J and K). These findings suggest that Ihh pathway is suppressed in endometrium of adenomyosis patients.

\section{LC3BII expression is elevated in endometrium of adenomyosis and endometriosis patients}

LC3 isastructural protein of theautophagosomemembrane and exists as four isoforms (LC3A, LC3B, LC3B2, and LC3C) in mammalian cells (Klionsky et al. 2016). LC3BII is converted from LC3BI by phosphatidylethanolamine conjugation and its expression correlates with autophagy activation (Mei et al. 2015, Felip et al. 2019). Therefore, we measured LC3BII levels by Western blot analysis as a surrogate marker for endometrial cell autophagy. In samples from endometriosis patients, we found that LC3BII expressions in eutopic and endometriotic tissues were nearly 1.4-fold and 2.6-fold of that in the control, respectively (Fig. 3A and C). Moreover, we observed that the LC3BII expression level in the eutopic tissues of adenomyosis patients was approximately 1.4 -fold of that in the controls (Fig. 3B and D). These results indicate that autophagy may be activated in the endometrium of women with endometriosis or adenomyosis.

\section{Decreased Ihh signaling activates autophagy in endometrial stromal cells}

To clarify the relationship between Ihh signaling and autophagy in endometrial cells, a small-molecule $\mathrm{Hh}$ signaling inhibitor GANT61, which directly blocks Gli-activated transcription, was employed in our in vitro experiments (Lauth et al. 2007). After GANT61 treatment, the expression level of LC3BII was found to be enhanced in EuESCs in a dose-dependent manner (Fig. 4A). Moreover, the expression of Beclin1, an essential protein in the autophagy interactome that signals the onset of autophagy (Klionsky et al. 2016), was significantly increased (Fig. 4A). The LC3 turnover assay was used to further evaluate the effects of GANT61 treatment on autophagy in EuESCs. 3-MA inhibits the initial steps of autophagosome formation, and CQ neutralizes lysosomal $\mathrm{pH}$ to block autophagolysosomal degradation. GANT61 treatment promoted LC3BII expression, whereas 3-MA significantly inhibited GANT61-induced LC3BII accumulation (Fig. 4B). In addition, LC3BII expression was elevated following treatment with CQ and GANT61 to a greater extent compared with treatment with either compound alone (Fig. 4B). Furthermore, we infected EuESCs with recombinant adenoviral vectors encoding a tandem sensor mCherry-eGFP-LC3B construct to monitor autophagic flux. eGFP is readily degraded under low $\mathrm{pH}$ and proteolytic conditions in the lysosome, whereas mCherry is more stable (Klionsky et al. 2016). Thus, yellow fluorescence generated by the colocalization of eGFP and mCherry indicates a phagophore or an autophagosome that has not fused with a lysosome (Klionsky et al. 2016). In contrast, autolysosomes only produce red signals (Klionsky et al. 2016). We observed that GANT61 $(10 \mu \mathrm{M})$ treatment significantly increased the number of red puncta in EuESCs (Fig. 4C and D). These results suggest that GANT61 treatment increases autophagy flux in EuESCs. To further confirm these results, we disrupted Ihh signaling by Gli1 knockdown. The results showed that Gli1 siRNA treatment suppressed Gli1 and Gli2 expression (Fig. 4E, F, G and H) but did not disrupt Ptch1 expression (data not shown). Importantly, we found that Gli1 knockdown significantly upregulated the expression of LC3BII and Beclin1 in EuESCs (Fig. 4G and H). The LC3 turnover assay showed that CQ treatment augmented Gli1 knockdown-induced LC3BII accumulation in EuESCs (Fig. 4I). Taken together, our data suggest that suppressed Ihh signaling activates autophagy in endometrial stromal cells. 

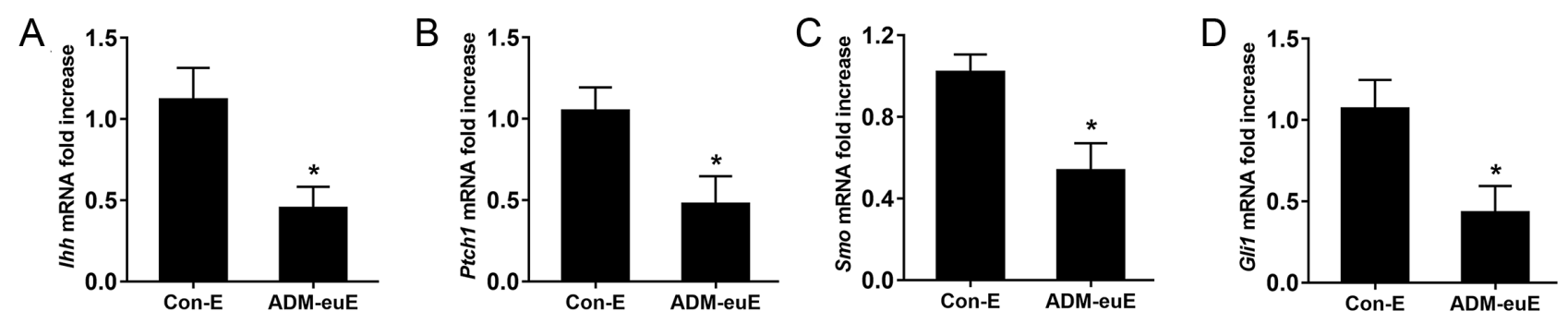

E

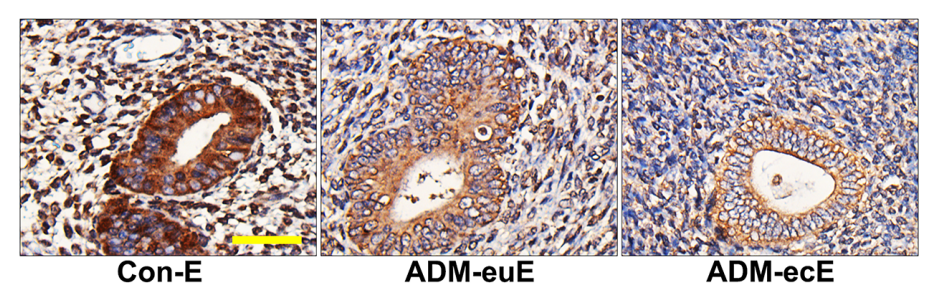

\section{G}
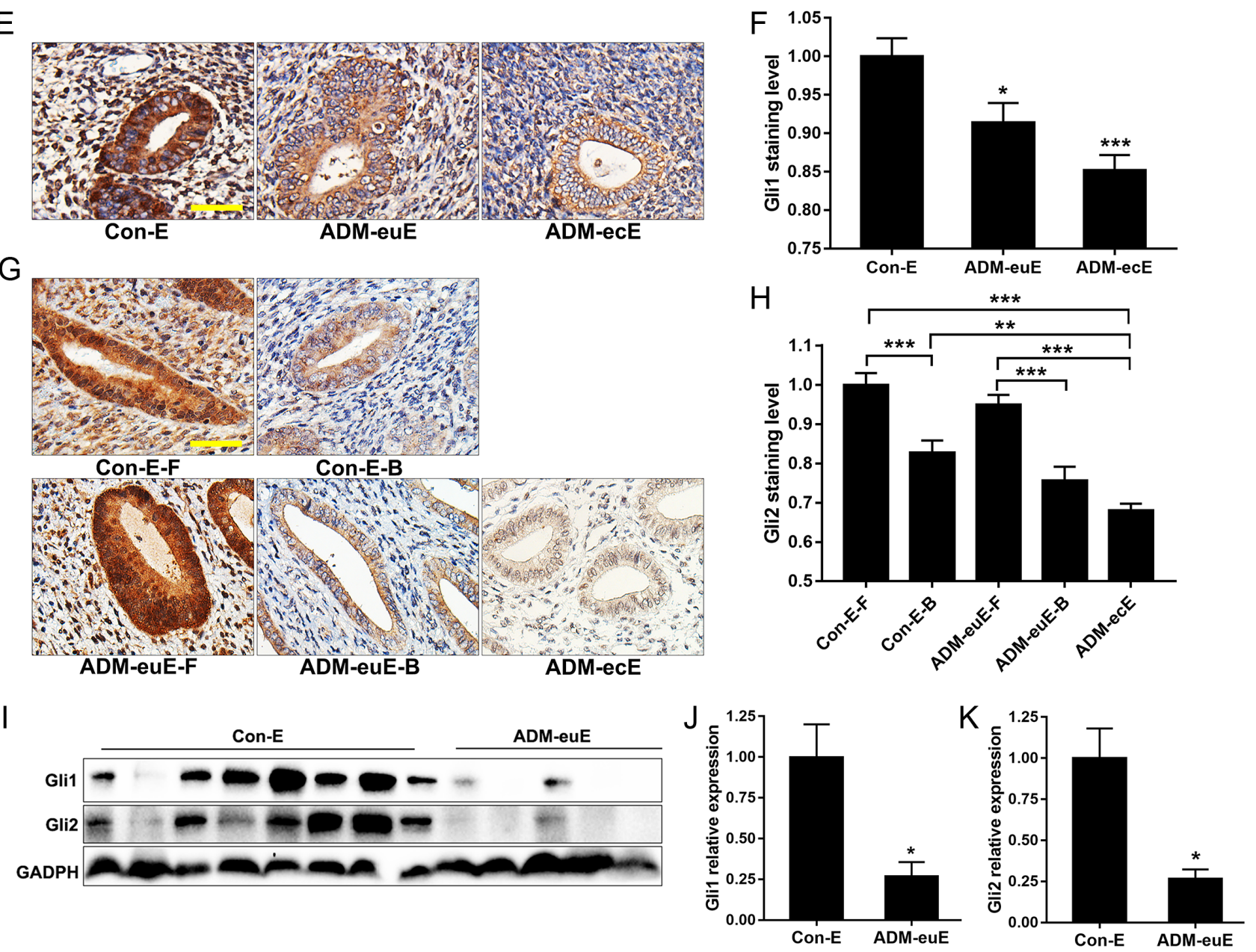

Figure 2 Ihh signaling is inhibited in endometrial tissues of women with adenomyosis. (A, B, C and D) Ihh, Ptch1, Smo and Gli1 mRNA expression levels were assessed via qPCR in Con-E and ADM-euE; $n=7$. (E and F) Representative immunohistochemical images of Gli1, and densitometric quantification of Gli1 staining levels in Con-E $(n=15)$, ADM-euE $(n=18)$ and ADM-ecE $(n=18)$. Scale bar $=50 \mu \mathrm{m}$. $(\mathrm{G}$ and H) Representative immunohistochemical images of Gli2, and densitometric quantification of Gli2 staining levels in Con-E ( $n=15)$, ADM-euE $(n=18)$ and ADM-ecE $(n=18)$. Scale bar $=50 \mu \mathrm{m}$. (I-K) Representative immunoblots and densitometric quantifications of Gli1 and Gli2 in Con-E $(n=8)$ and ADM-euE $(n=5)$. Quantitative results are expressed as means \pm S.E.M. ${ }^{*} P<0.05,{ }^{* *} P<0.01,{ }^{* * *} P<0.001$. Con-E: endometrium from patients with leiomyoma; ADM-euE and ADM-ecE: eutopic and ectopic endometrial tissues from adenomyosis patients, respectively.

\section{Suppressed Ihh signaling promotes cell survival via autophagy}

It is unknown whether autophagy is beneficial or detrimental to the survival of endometriotic cells. Previous reports indicate that ectopic endometrial cells in endometriotic ovarian cysts may experience strong and persistent oxidative stress (Yamaguchi et al. 2008, Sanchez et al. 2014, Allavena et al. 2015). Therefore, we used $\mathrm{H}_{2} \mathrm{O}_{2}$ to induce intracellular oxidative stress to determine the role of autophagy in EuESCs. GANT61 $(0-20 \mu \mathrm{M})$ did not affect the viability of EuESCs over $24 \mathrm{~h}$ (Fig. 5A). The LC3 turnover assay showed that LC3BII production in response to $2 \mathrm{~h}$ of $\mathrm{H}_{2} \mathrm{O}_{2}(200$ $\mu \mathrm{M})$ treatment after CQ $(10 \mu \mathrm{M})$ preincubation for 24 $\mathrm{h}$ was significantly increased compared with that in the control, $\mathrm{CQ}$, or $\mathrm{H}_{2} \mathrm{O}_{2}$ treatment groups, indicating that 


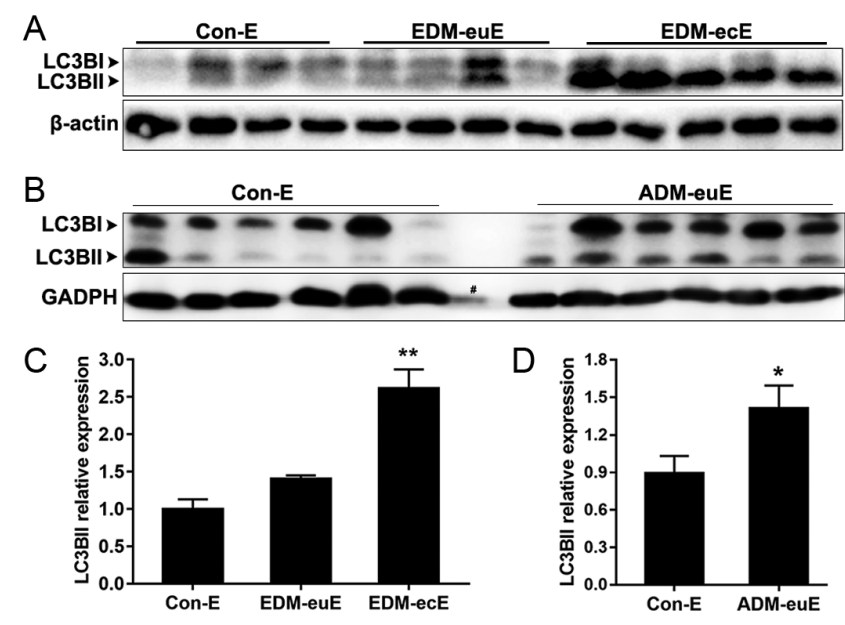

Figure 3 LC3BII expression is elevated in endometrium of adenomyosis and endometriosis patients. (A and C) Representative immunoblots and densitometric quantifications of LC3BII levels in Con-E $(n=8)$, EDM-euE $(n=10)$ and EDM-ecE $(n=11)$. (B and D) Representative immunoblots and densitometric quantifications of LC3BII levels in Con-E $(n=12)$ and ADM-euE $(n=10)$. Quantitative results are expressed as means \pm S.E.M. ${ }^{*} P<0.05,{ }^{*} P<0.01$. \#Nonspecific band. Con-E: endometrium from patient with leiomyoma; EDM-euE and EDM-ecE: eutopic and ectopic endometrial tissues from endometriosis patients, respectively. ADM-euE: eutopic endometrial tissues from adenomyosis patients.

$\mathrm{H}_{2} \mathrm{O}_{2}$ may activate autophagy in EuESCs (Fig. 5B). In addition, we found that $2 \mathrm{~h}$ of $\mathrm{H}_{2} \mathrm{O}_{2}$ exposure following pretreatment with GANT61 $(10 \mu \mathrm{M})$ and CQ for $24 \mathrm{~h}$ significantly augmented LC3BII accumulation compared with $\mathrm{H}_{2} \mathrm{O}_{2}$ incubation after GANT61 pretreatment. This suggests that oxidative stress may increase Hh signaling inhibition-induced autophagic flux in EuESCs (Fig. 5B). The extent of nuclear condensation in EuESCs following exposure to $\mathrm{H}_{2} \mathrm{O}_{2}$ for $2 \mathrm{~h}$ was assessed by DAPI staining. The number of pyknotic nuclei was significantly increased following $\mathrm{H}_{2} \mathrm{O}_{2}$ treatment (Fig. 5C, white arrowheads; and Fig. 5D). GANT61 treatment decreased the number of $\mathrm{H}_{2} \mathrm{O}_{2}$-induced pyknotic nuclei, whereas $\mathrm{CQ}$ treatment diminished GANT61-induced cell protection in $\mathrm{H}_{2} \mathrm{O}_{2}$-stressed EuESCs (Fig. 5C and D). The results suggest that autophagy activated by Hh signaling inhibition may contribute to increased cell survival under oxidative stress in ectopic sites.

\section{Discussion}

A previously reported study has demonstrated that many upstream molecular aberrations such as KRAS activation and overexpression of SIRT1/BCL6 in the endometrium (Yoo et al. 2017), may disrupt endometrial Ihh signaling. In this study, we found that the Ihh signaling was significantly inhibited in endometrial tissues collected from women with endometriosis, which is consistent with several previous reports (Smith et al. 2011, Yoo et al. 2017). The same alteration of Ihh signaling was also observed in the proliferative phase of tissues isolated from adenomyosis patients. Given that Ihh signaling plays an important role in the progesterone reaction, impaired Ihh signaling may result in progesterone resistance and contribute to infertility in women with endometriosis or adenomyosis (Lee et al. 2006, Smith et al. 2011, Yoo et al. 2017). Endometriosis and adenomyosis are viewed as estrogen-dependent diseases and are associated with increased local estrogen levels (Vannuccini et al. 2017, Zondervan et al. 2020). Moreover, fibrosis generally occurs in ectopic lesions during adenomyosis development (Shen et al. 2016, Vannuccini et al. 2017, Kobayashi et al. 2020). It has been reported that conditional ablation of $/ h h$ stimulated estrogen signaling and enhanced collagen production in the murine uterus (Franco et al. 2010). This indicates that Ihh signaling inhibition may be implicated in local aberrant estrogen activity and fibrogenesis in eutopic or ectopic endometrium of endometriosis or adenomyosis patients.

Immunohistochemical analysis demonstrated that the distribution of endometrial Gli2 was spatially different as its expression was markedly increased in the functional layer compared with the basal endometrium layer. Gli2 functions as a transcriptional activator of Hh signaling pathway (Jimenez-Sanchez et al. 2012). Therefore, Ihh signaling in the functional endometrial layer may be more active, which implies that the progesterone signaling in the functional endometrium layer may be more active than that in the basal endometrium layer.

Many reports have indicated that autophagy may be decreased in endometrial tissues from endometriosis or adenomyosis patients (Ren et al. 2010, Mei et al. 2015, Zhan et al. 2018). However, evidence has also demonstrated that endometrial autophagy may be upregulated in the endometria of women with endometriosis (Allavena et al. 2015, Liu et al. 2017, 2019b, Zheng et al. 2018). In this study, we evaluated the autophagy activity by assessing the most widely used marker, LC3BII (Klionsky et al. 2016), and confirmed that autophagy may be activated in endometrial tissues of women with endometriosis or adenomyosis. Autophagy frequently occurs in response to various stimuli, including starvation, endoplasmic reticulum stress, hypoxia, and oxidative stress (Allavena et al. 2015, Liu et al. 2017, Zhang et al. 2020). In fact, previous reports have demonstrated that endometriotic tissues may experience hypoxia in early implanted ectopic endometrial lesions and suffer overwhelming oxidative stress in old ectopic lesions (Yamaguchi et al. 2008, Allavena et al. 2015, Xiong et al. 2016).

The Hh pathway may be primarily considered an inhibitory cell-signaling with respect to autophagy (Jimenez-Sanchez et al. 2012). However, whether decreased Ihh signaling in the endometrium promotes autophagy remains to be determined. Our results demonstrated that suppression of Ihh signaling either 

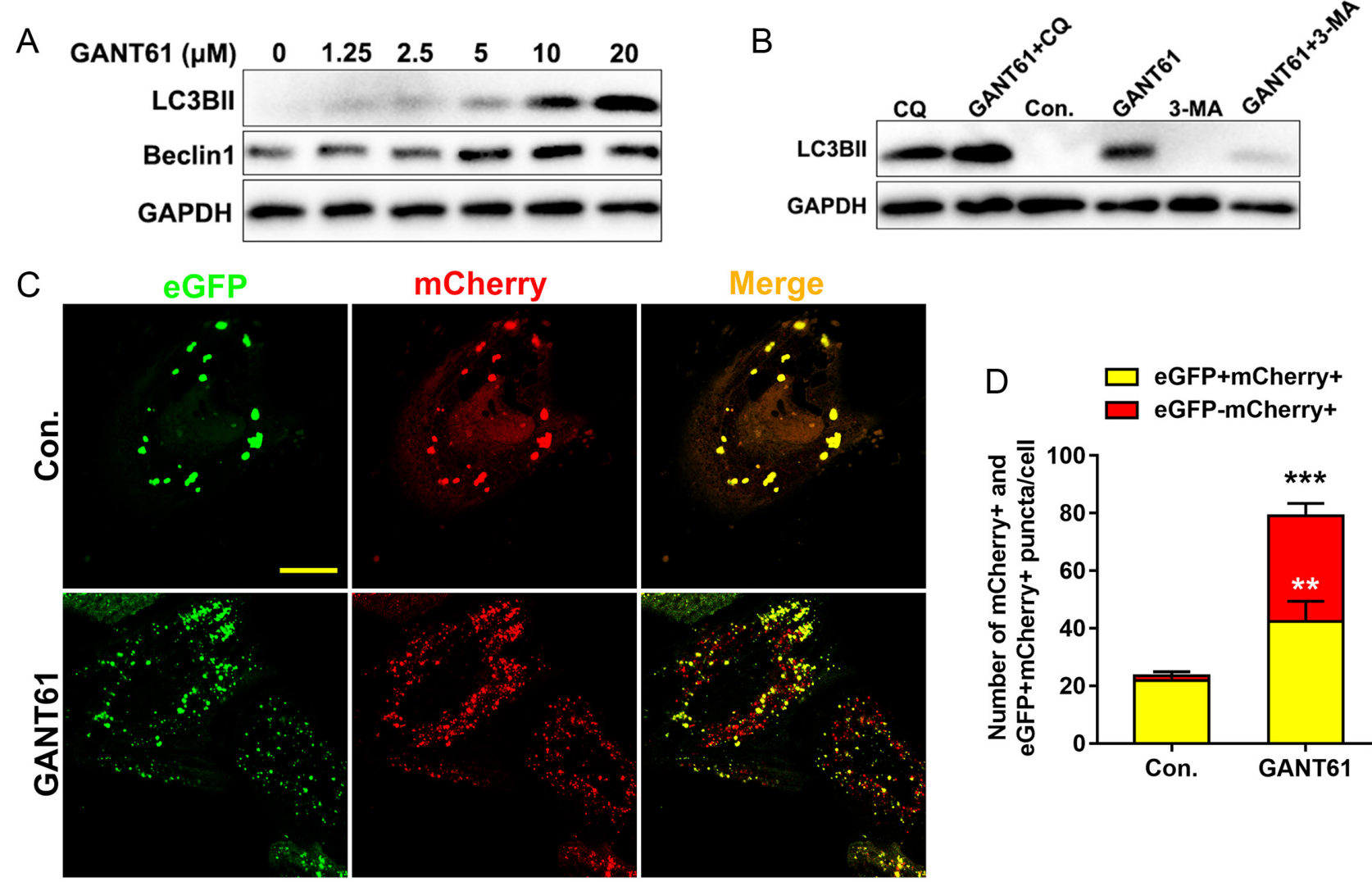

$\mathrm{E}$

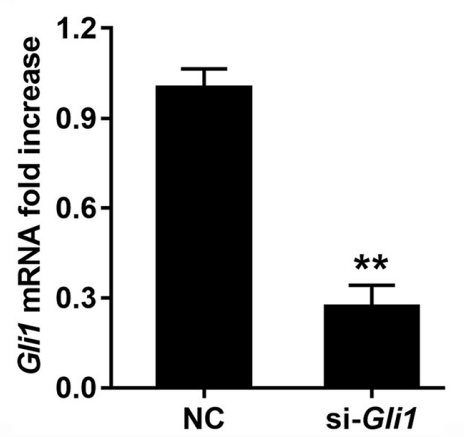

F

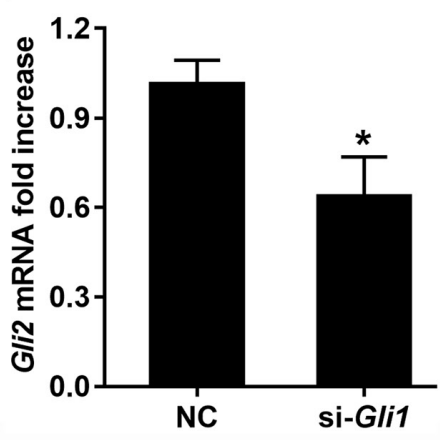

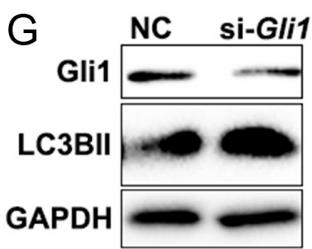
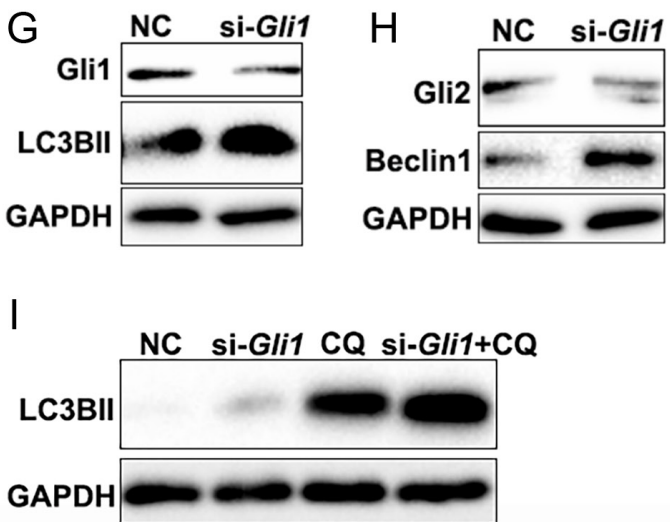

Figure 4 Decreased Ihh signaling activates autophagy in endometrial stromal cells. (A) LC3BII and Beclin1 protein levels were measured by Western blot after EuESCs were treated with GANT61 (0-20 $\mu \mathrm{M})$ for $24 \mathrm{~h}$. (B) LC3BII levels were measured by Western blot after EuESCs were treated with $10 \mu \mathrm{M}$ GANT61 for $24 \mathrm{~h}$ in the absence or presence of $2.5 \mathrm{mM} 3-\mathrm{MA}$ or $10 \mu \mathrm{M} \mathrm{CQ}$. (C) Recombinant adenovirus vectors encoding mCherry-eGFP-LC3B were incubated with EuESCs for $24 \mathrm{~h}$. The cells were then treated with $10 \mu \mathrm{M}$ GANT61 for $24 \mathrm{~h}$ and analyzed by laser scanning confocal microscopy. Con., control; scale bar $=20 \mu \mathrm{m}$. (D) Quantification of mCherry+eGFP- (red) and mCherry+eGFP+ (yellow) signals (puncta). Quantitative results are expressed as means \pm s.E.M. of at least four independent experiments. ${ }^{* *} P<0.01,{ }^{* * *} P<0.001$. (E, $\mathrm{F}$, $\mathrm{G}, \mathrm{H}$ and I) EuESCs were transfected with siRNA targeting Gli1 and received CQ treatment as described in the 'Materials and methods' section, Gli1, Gli2, LC3BII, and Beclin1 levels were then evaluated with qPCR and/or Western blot. Quantitative results are expressed as means \pm S.E.M. of at least five independent experiments. ${ }^{*} P<0.05,{ }^{* *} P<0.01$.

with the small-molecule inhibitor, GANT61, or by Gli1 silencing markedly enhanced autophagic flux in endometrial stromal cells. These results, with the increased endometrial LC3BII expression observed in eutopic and ectopic tissues, indicate that inhibition of endometrial thh signaling contributes to the functional activation of autophagy in endometrial tissues from endometriosis and adenomyosis patients.

Repeated monthly hemorrhage during endometriosis and adenomyosis causes high levels of local catalytic ferrous iron deposition in ectopic lesions, which can generate a large number of hydroxyl radicals via the 


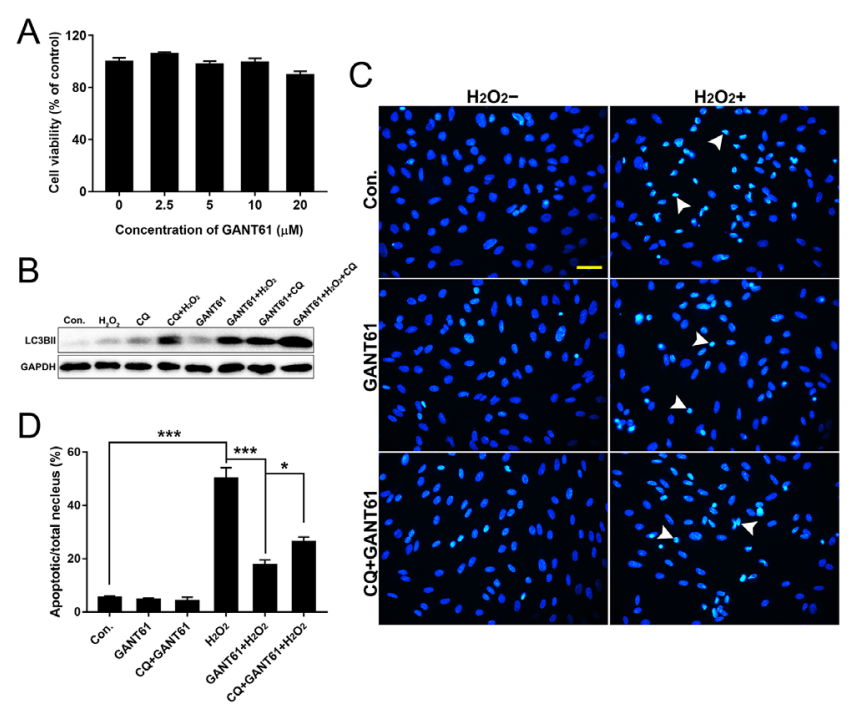

Figure 5 Suppressed Ihh signaling promotes cell survival via autophagy. (A) EuESCs were treated with GANT61 (0-20 $\mu \mathrm{M})$ for 24 h. Cell viability was evaluated with the Cell Counting Kit-8 $(n=6)$. (B) After EuESCs were treated with GANT61 $(10 \mu \mathrm{M})$ alone or in combination with CQ $(10 \mu \mathrm{M})$ for $24 \mathrm{~h}$, cells were exposed to 200 $\mu \mathrm{M} \mathrm{H}_{2} \mathrm{O}_{2}$ for $2 \mathrm{~h}$. Then, LC3BII protein levels were measured by Western blot. (C) Morphological changes in the apoptosis-like nuclei in EuESCs were evaluated by DAPI staining; white arrowheads indicate pyknotic nuclei that underwent apoptosis. Scale bar $=50$ $\mu \mathrm{m}$; Con., control. (D) The ratio of apoptotic nuclei to total nuclei was calculated and expressed as the apoptosis rate; each value is presented as the mean \pm S.E.M. of at least five fields of view under the microscope. ${ }^{*} P<0.05,{ }^{* * *} P<0.001$; Con., control.

Fenton reaction (Yamaguchi et al. 2008, Mori et al. 2015). This may contribute to oxidative stress in the local microenvironment of ectopic tissues. For ectopic endometrial cells immersed in the local fluid environment, the most challenging objective is to survive. However, the underlying mechanism of survival has not been fully elucidated. Autophagy is generally regarded as a beneficial mechanism for cell survival (Leidal et al. 2018, Mizushima 2018). Our study demonstrated that GANT61-induced autophagy significantly decreased oxidative stress-induced cell apoptosis, indicating that Ihh signaling inhibition may restore intracellular homeostasis through autophagy in ectopic endometrial cells. Our study provides experimental evidence to support the hypothesis that endometrial autophagy activation in endometriosis or adenomyosis may be regarded as an adaptive response that contributes to the survival of endometrial cells in ectopic sites as well as lesion maintenance (Allavena et al. 2015). However, this hypothesis requires further validation in animal models.

Collectively, as depicted in Fig. 6, our study demonstrated that Ihh signaling is inhibited in endometriosis and adenomyosis patients, and this decrease may be implicated in the activation of prosurvival autophagy in ectopic tissues. The current study
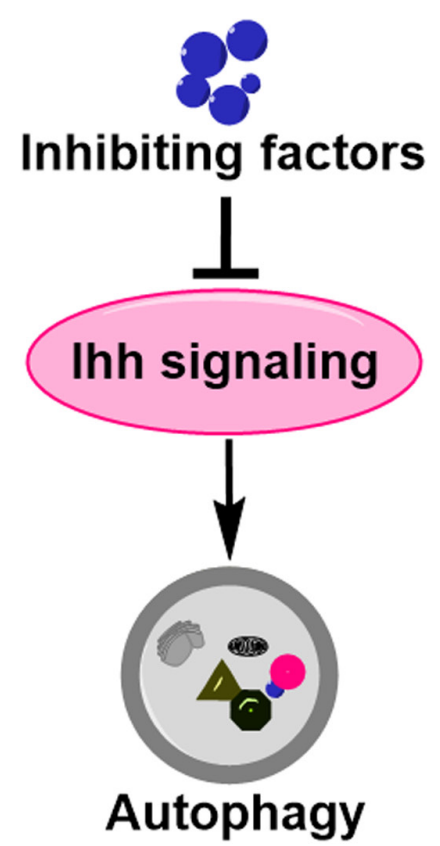

$\downarrow$ Activation

Inhibition

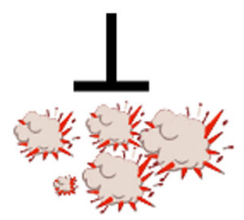

Oxidative stressinduced cell death

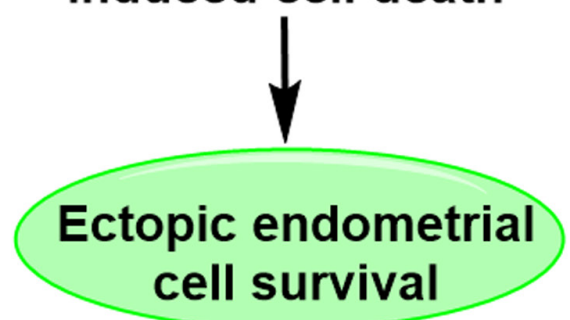

Figure 6 Proposed model for the roles of Ihh signaling and autophagy in the pathogenesis of endometriosis and adenomyosis. Various kinds of inhibiting factors can disrupt lhh signaling in the endometrium. Decreased Ihh signaling increases autophagic activity in ectopic endometria of endometriosis and adenomyosis patients. Autophagy may decrease oxidative stress-induced cell death in ectopic lesions, thus promoting the survival of endometriotic cells in ectopic sites and contributing to lesion maintenance.

expands our understanding of the roles of thh signaling and autophagy in the pathogenesis of endometriosis and adenomyosis and may lead to new therapeutic interventions.

\section{Declaration of interest}

The authors declare that there is no conflict of interest that could be perceived as prejudicing the impartiality of the research reported. 


\section{Funding}

The present study was supported by the National Natural Science Foundation of China (grant number 81801424), Basic Public Welfare Research Project of Zhejiang Province (grant number LGF19H060001), and the Scientific Research Project of Taizhou Science and Technology Bureau in Zhejiang Province (grant number 1801 ky60).

\section{Ethical approval and informed consent}

This study was approved by the local ethical committee and the written informed consent was obtained from all patients.

\section{Author contribution statement}

Y Z, Y P and Q X performed Western blot analysis, immunohistochemical staining and cell experiments. $L Z$ and Y C carried out qPCR analysis. D Y and H Z collected tissues and contributed critical discussions. $X Z$ and $J \mathrm{~L}$ designed the study, analyzed the data and wrote the manuscript. All authors approved the final manuscript.

\section{References}

Allavena G, Carrarelli P, Del Bello B, Luisi S, Petraglia F \& Maellaro E 2015 Autophagy is upregulated in ovarian endometriosis: a possible interplay with p53 and heme oxygenase-1. Fertility and Sterility 103 1244.e11251.e1. (https://doi.org/10.1016/j.fertnstert.2015.02.007)

Che X, Wang J, He J, Yu Q, Sun W, Chen S, Zou G, Li T, Guo X \& Zhang X 2020 A new trick for an old dog: the application of mifepristone in the treatment of adenomyosis. Journal of Cellular and Molecular Medicine 24 1724-1737. (https://doi.org/10.1111/jcmm.14866)

Choi J, Jo M, Lee E, Lee DY \& Choi D 2015 Dienogest enhances autophagy induction in endometriotic cells by impairing activation of AKT, ERK1/2, and mTOR. Fertility and Sterility 104 655-664. (https://doi.org/10.1016/j. fertnstert.2015.05.020)

Dai P, Akimaru H, Tanaka Y, Maekawa T, Nakafuku M \& Ishii S 1999 Sonic hedgehog-induced activation of the Gli1 promoter is mediated by GLI3. Journal of Biological Chemistry 274 8143-8152. (https://doi. org/10.1074/jbc.274.12.8143)

Felip I, Moiola CP, Megino-Luque C, Lopez-Gil C, Cabrera S, SoleSanchez S, Munoz-Guardiola P, Megias-Roda E, Perez-Montoyo $H$, Alfon J et al. 2019 Therapeutic potential of the new TRIB3-mediated cell autophagy anticancer drug ABTL0812 in endometrial cancer. Gynecologic Oncology 153 425-435. (https://doi.org/10.1016/j. ygyno.2019.03.002)

Franco HL, Lee KY, Broaddus RR, White LD, Lanske B, Lydon JP, Jeong JW \& DeMayo FJ 2010 Ablation of Indian hedgehog in the murine uterus results in decreased cell cycle progression, aberrant epidermal growth factor signaling, and increased estrogen signaling. Biology of Reproduction 82 783-790. (https://doi.org/10.1095/ biolreprod.109.080259)

Garcia-Solares J, Donnez J, Donnez O \& Dolmans MM 2018 Pathogenesis of uterine adenomyosis: invagination or metaplasia? Fertility and Sterility 109 371-379. (https://doi.org/10.1016/j.fertnstert.2017.12.030)

Gorojankina T 2016 Hedgehog signaling pathway: a novel model and molecular mechanisms of signal transduction. Cellular and Molecular Life Sciences 73 1317-1332. (https://doi.org/10.1007/s00018-0152127-4)

He Y, Guo Q, Cheng Y, Qu Y, Sun L, Kong C, Lei L \& Zhang G 2018 Abnormal activation of the sonic hedgehog signaling pathway in endometriosis and its diagnostic potency. Fertility and Sterility 110128. e2-136.e2. (https://doi.org/10.1016/j.fertnstert.2018.02.138)

Jimenez-Sanchez M, Menzies FM, Chang YY, Simecek N, Neufeld TP \& Rubinsztein DC 2012 The Hedgehog signalling pathway regulates autophagy. Nature Communications 3 1200. (https://doi.org/10.1038/ ncomms2212)

Klionsky DJ, Abdelmohsen K, Abe A, Abedin MJ, Abeliovich H, Acevedo Arozena A, Adachi H, Adams CM, Adams PD, Adeli K et al. 2016 Guidelines for the use and interpretation of assays for monitoring autophagy (3rd edition). Autophagy 12 1-222. (https://doi.org/10.1080/ 15548627.2015.1100356)

Kobayashi H, Kishi Y \& Matsubara S 2020 Mechanisms underlying adenomyosis-related fibrogenesis. Gynecologic and Obstetric Investigation 85 1-12. (https://doi.org/10.1159/000502822)

Lauth M, Bergstrom A, Shimokawa T \& Toftgard R 2007 Inhibition of GLI-mediated transcription and tumor cell growth by smallmolecule antagonists. PNAS 104 8455-8460. (https://doi.org/10.1073/ pnas.0609699104)

Lee K, Jeong J, Kwak I, Yu CT, Lanske B, Soegiarto DW, Toftgard R, Tsai MJ, Tsai S, Lydon JP et al. 2006 Indian hedgehog is a major mediator of progesterone signaling in the mouse uterus. Nature Genetics 38 1204-1209. (https://doi.org/10.1038/ng1874)

Leidal AM, Levine B \& Debnath J 2018 Autophagy and the cell biology of age-related disease. Nature Cell Biology 20 1338-1348. (https://doi. org/10.1038/s41556-018-0235-8)

Li H, Li J, Li Y, Singh P, Cao L, Xu LJ, Li D, Wang Y, Xie Z, Gui Y et al. 2012 Sonic hedgehog promotes autophagy of vascular smooth muscle cells. American Journal of Physiology: Heart and Circulatory Physiology 303 H1319-H1331. (https://doi.org/10.1152/ajpheart.00160.2012)

Liu H, Zhang Z, Xiong W, Zhang L, Xiong Y, Li N, He H, Du Y \& Liu Y 2017 Hypoxia-inducible factor-1alpha promotes endometrial stromal cells migration and invasion by upregulating autophagy in endometriosis. Reproduction 153 809-820. (https://doi.org/10.1530/REP-16-0643)

Liu H, Zhang W, Wang L, Zhang Z, Xiong W, Zhang L, Fu T, Li X, Chen Y \& Liu Y 2019a GLI1 is increased in ovarian endometriosis and regulates migration, invasion and proliferation of human endometrial stromal cells in endometriosis. Annals of Translational Medicine 7 663. (https://doi. org/10.21037/atm.2019.10.76)

Liu H, Zhang Z, Xiong W, Zhang L, Du Y, Liu Y \& Xiong X 2019b Long noncoding RNA MALAT1 mediates hypoxia-induced pro-survival autophagy of endometrial stromal cells in endometriosis. Journal of Cellular and Molecular Medicine 23 439-452. (https://doi.org/10.1111/jcmm.13947)

Livak KJ \& Schmittgen TD 2001 Analysis of relative gene expression data using real-time quantitative PCR and the 2(-Delta Delta C(T)) Method. Methods 25 402-408. (https://doi.org/10.1006/meth.2001.1262)

Matsuzaki S, Pouly JL \& Canis M 2018 In vitro and in vivo effects of MK2206 and chloroquine combination therapy on endometriosis: autophagy may be required for regrowth of endometriosis. British Journal of Pharmacology 175 1637-1653. (https://doi.org/10.1111/bph.14170)

Mei J, Zhu XY, Jin LP, Duan ZL, Li DJ \& Li MQ 2015 Estrogen promotes the survival of human secretory phase endometrial stromal cells via CXCL12/CXCR4 up-regulation-mediated autophagy inhibition. Human Reproduction 30 1677-1689. (https://doi.org/10.1093/humrep/dev100)

Mizushima N 2018 A brief history of autophagy from cell biology to physiology and disease. Nature Cell Biology 20 521-527. (https://doi. org/10.1038/s41556-018-0092-5)

Mizushima N, Yoshimori T \& Levine B 2010 Methods in mammalian autophagy research. Cell 140 313-326. (https://doi.org/10.1016/j. cell.2010.01.028)

Mori M, Ito F, Shi L, Wang Y, Ishida C, Hattori Y, Niwa M, Hirayama T, Nagasawa H, Iwase A et al. 2015 Ovarian endometriosis-associated stromal cells reveal persistently high affinity for iron. Redox Biology 6 578-586. (https://doi.org/10.1016/j.redox.2015.10.001)

Petralia RS, Schwartz CM, Wang YX, Kawamoto EM, Mattson MP \& Yao PJ 2013 Sonic hedgehog promotes autophagy in hippocampal neurons. Biology Open 2 499-504. (https://doi.org/10.1242/bio.20134275)

Ren Y, Mu L, Ding X \& Zheng W 2010 Decreased expression of Beclin 1 in eutopic endometrium of women with adenomyosis. Archives of Gynecology and Obstetrics 282 401-406. (https://doi.org/10.1007/ s00404-009-1280-0)

Ruiz I Altaba A 1999 Gli proteins encode context-dependent positive and negative functions: implications for development and disease. Development 126 3205-3216.

Sanchez AM, Vigano P, Somigliana E, Panina-Bordignon P, Vercellini P \& Candiani M 2014 The distinguishing cellular and molecular features of the endometriotic ovarian cyst: from pathophysiology to the potential 
endometrioma-mediated damage to the ovary. Human Reproduction Update 20 217-230. (https://doi.org/10.1093/humupd/dmt053)

Shen M, Liu X, Zhang H \& Guo SW 2016 Transforming growth factor beta1 signaling coincides with epithelial-mesenchymal transition and fibroblast-to-myofibroblast transdifferentiation in the development of adenomyosis in mice. Human Reproduction 31 355-369. (https://doi. org/10.1093/humrep/dev314)

Shi Y \& Long F 2017 Hedgehog signaling via Gli2 prevents obesity induced by high-fat diet in adult mice. elife $\mathbf{6}$ e31649. (https://doi.org/10.7554/ eLife.31649)

Smith K, Alnifaidy R, Wei Q \& Nieman LK 2011 Endometrial Indian hedgehog expression is decreased in women with endometriosis. Fertility and Sterility 95 2738.e1-2741.e1. (https://doi.org/10.1016/j. fertnstert.2011.05.018)

Vannuccini S, Tosti C, Carmona F, Huang SJ, Chapron C, Guo SW \& Petraglia F 2017 Pathogenesis of adenomyosis: an update on molecular mechanisms. Reproductive Biomedicine Online 35 592-601. (https:// doi.org/10.1016/j.rbmo.2017.06.016)

Wang Y, Han C, Lu L, Magliato S \& Wu T 2013 Hedgehog signaling pathway regulates autophagy in human hepatocellular carcinoma cells. Hepatology 58 995-1010. (https://doi.org/10.1002/hep.26394)

Xiong Y, Liu Y, Xiong W, Zhang L, Liu H, Du Y \& Li N 2016 Hypoxiainducible factor 1alpha-induced epithelial-mesenchymal transition of endometrial epithelial cells may contribute to the development of endometriosis. Human Reproduction 31 1327-1338. (https://doi. org/10.1093/humrep/dew081)

Yamaguchi K, Mandai M, Toyokuni S, Hamanishi J, Higuchi T, Takakura K \& Fujii S 2008 Contents of endometriotic cysts, especially the high concentration of free iron, are a possible cause of carcinogenesis in the cysts through the iron-induced persistent oxidative stress. Clinical Cancer Research 14 32-40. (https://doi.org/10.1158/1078-0432.CCR-07-1614)

Yim WW \& Mizushima N 2020 Lysosome biology in autophagy. Cell Discovery 6 6. (https://doi.org/10.1038/s41421-020-0141-7)
Yoo JY, Kim TH, Fazleabas AT, Palomino WA, Ahn SH, Tayade C, Schammel DP, Young SL, Jeong JW \& Lessey BA 2017 KRAS activation and over-expression of SIRT1/BCL6 contributes to the pathogenesis of endometriosis and progesterone resistance. Scientific Reports 76765. (https://doi.org/10.1038/s41598-017-04577-w)

Zhan L, Yao S, Sun S, Su Q, Li J \& Wei B 2018 NLRC5 and autophagy combined as possible predictors in patients with endometriosis. Fertility and Sterility $\mathbf{1 1 0}$ 949-956. (https://doi.org/10.1016/j. fertnstert.2018.06.028)

Zhang L, Zhou Y, Xia Q, Chen Y \& Li J 2020 All-trans-retinal induces autophagic cell death via oxidative stress and the endoplasmic reticulum stress pathway in human retinal pigment epithelial cells. Toxicology Letters 322 77-86. (https://doi.org/10.1016/j.toxlet.2020.01.005)

Zheng J, Luo X, Bao J, Huang X, Jin Y, Chen L \& Zheng F 2018 Decreased expression of HOXA10 may activate the autophagic process in ovarian endometriosis. Reproductive Sciences 25 1446-1454. (https://doi. org/10.1177/1933719118768704)

Zhu B, Chen Y, Shen X, Liu X \& Guo SW 2016 Anti-platelet therapy holds promises in treating adenomyosis: experimental evidence. Reproductive Biology and Endocrinology 14 66. (https://doi.org/10.1186/s12958-0160198-1)

Zondervan KT, Becker CM \& Missmer SA 2020 Endometriosis. New England Journal of Medicine 382 1244-1256. (https://doi.org/10.1056/ NEJMra1810764)

Received 25 March 2020

First decision 30 April 2020

Revised Manuscript received 14 October 2020

Accepted 17 November 2020 